Objectives This study assessed the impact of 1) within-individual improvements, and 2) within-individual deteriorations in working conditions, health behaviour and BMI on changes in work ability and self-rated health among workers.

Methods The Dutch Study on Transitions in Employment, Ability and Motivation (STREAM) was used to identify participants whose working conditions, health behaviour, and BMI improved $(\mathrm{N}=14,045)$ or deteriorated $(\mathrm{N}=14,066)$ at least once during seven year follow-up (2010-2017). The impact of within-individual improvements and deteriorations in health behaviour (moderate- and vigorous physical activity, smoking status), BMI, psychosocial (psychological- and emotional job demands, autonomy, social support) and physical working conditions in a given year on changes in work ability (0-10 scale) and self-rated health (1-5 scale) in the same year were analysed with fixed-effects regression models.

Results Workers with deteriorated physical or psychosocial working conditions decreased in work ability ( $\beta$ 's: $-0.21(95 \%$ CI: $-0.25 ;-0.18)$ to $-0.28(95 \% \mathrm{CI}:-0.33 ;-0.24))$ and health ( $\beta$ 's: -0.07 (95\%CI: $-0.09 ;-0.06)$ to -0.10 (95\%CI: -0.12 ;$0.08)$ ), whereas improvements in working conditions were to a lesser extent associated with increased work ability ( $\beta$ 's: 0.06 (95\%CI: $0.02 ; 0.09)$ to 0.11 (95\%CI: $0.06 ; 0.16)$ ) and health ( $\beta$ 's: $0.02 \quad(95 \% \mathrm{CI}: 0.00 ; 0.03)$ to $0.04 \quad(95 \% \mathrm{CI}$ : $0.02 ; 0.06))$. Workers with increased BMI or decreased physical activity had reduced work ability and health. Likewise, decreased BMI or increased vigorous physical activity was associated with improved health. An increase in moderate or vigorous physical activity was modestly associated with a reduced work ability. Quitting smoking was associated with reduced work ability and health.

Conclusion Preventing deteriorations in working conditions, health behaviour and BMI could be of importance for sustainable employability.

\section{0-357 EXAMINING VARIATIONS IN WORK DISABILITY DURATION BY FIRM SIZE: A COMPARATIVE STUDY OF WORKERS' COMPENSATION CLAIMS IN CANADA AND AUSTRALIA}

Robert Macpherson, Tyler Lane, Alex Collie, 'Chris McLeod. 'University of British Columbia, Canada

\subsection{6/OEM-2021-EPI.152}

Introduction Small firms, while more numerous than large firms, often face greater challenges in implementing effective occupational health and safety and return-to-work programs. Research has rarely looked at firm size as a determinant of work disability duration and has been limited to single jurisdictions.

Objectives To identify whether there were differences in work disability duration between injured workers employed by small, medium and large firms and whether these differences varied between workers' compensation jurisdictions in Canada (CAN) and Australia (AUS).

Methods Workers' compensation data were used to identify comparable lost-time, work-related injury and musculoskeletal disorder claims in five Canadian and five Australian jurisdictions between 2011 and 2015. Work disability duration was measured using cumulative days in receipt of disability benefit payments up to one-year post-injury. Jurisdiction-specific quantile regression models were used to compare cumulative disability days paid from small $(<20$ full-time equivalents (FTEs), medium (20-199 FTEs), large (200+ FTEs) firms at 25th, 50th, and 75th percentiles in the disability distribution, adjusting for confounders.

Results Differences in work disability duration by firm size were observed in all jurisdictions except the Northern Territories (AUS). Compared to large firms, small firms were paid the most disability days at each percentile, particunarly in Victoria (AUS), Saskatchewan (CAN), the Australian Capital Territory, and Tasmania (AUS), where an additional 63.0, 31.1, 37.0 , and 27.4 days were paid at the 75 th percentiles of the distributions, respectively. Claims from medium-sized firms were generally paid more disability days than large firms except in Western Australia and Tasmania, where they were paid less.

Conclusions Small firms were shown to have the longest work disability durations in 9 of the 10 study jurisdictions. Claims management processes need to be sensitive to the challenges that small firms face in accommodating and returning injured workers back to work.

\section{Oral}

\section{0-366 VACCINE HESITANCY AMONG CANADIAN PARAMEDICS DURING THE COVID-19 PANDEMIC}

'David O'Neill, Tracy Kirkham, Paul Demers, Christopher MacDonald, Brian Grunau, Julie Bettinger, David Goldfarb, Jennie Helmer. 'University of Toronto, Canada

\subsection{6/OEM-2021-EPI.153}

Introduction Paramedics may be at an increased risk of interacting with COVID-19-positive individuals, making understanding the factors that influence paramedics' vaccination decisions increasingly important.

Objectives We aim to investigate factors that may influence paramedics' likelihood of COVID-19 vaccination.

Methods Canadian paramedics from five provinces (Alberta, British Columbia, Manitoba, Ontario, Saskatchewan) working during the COVID-19 pandemic were voluntarily recruited through posters, social media, and emails from collaborating paramedic organizations. Participants completed online questionnaires between January and May of 2021 that assessed COVID-19 vaccine status, vaccine hesitancy, and intent to be vaccinated. Differences in proportions tests were used to compare agreement scores, calculated by combining proportions of participants who responded 'strongly agree' and 'agree' to questionnaire items.

Results Of the 2178 paramedics recruited, 95.7\% completed the questionnaire $(76.6 \%$ vaccinated). While most participants $(89.4 \%)$ agreed that people should be vaccinated against COVID-19 and that vaccinations are necessary (94.7\%), fewer participants agreed that COVID-19 vaccines are safe $(78.5 \%)$ as compared to routine vaccines $(86.1 \%, \mathrm{p}<0.001)$, such as influenza vaccinations. However, vaccinated participants were more likely than unvaccinated participants to agree that routine vaccines are safe $(90.5 \%$ vs. $76.2 \%, \mathrm{p}<0.001)$ and that COVID-19 vaccines are safe $(87.3 \%$ vs. $52.4 \%, \mathrm{p}<0.001)$. Unvaccinated participants were more likely than vaccinated participants to report no intention of being vaccinated $(14.2 \%$ vs. $0.1 \%, \mathrm{p}<0.001)$, to report that they would get vaccinated but would wait $(22.5 \%$ vs. $9.4 \%, \mathrm{p}<0.001)$, and to report 